\title{
Gastric Interposition Following Transhiatal Esophagectomy: Radiographic Evaluation
}

\author{
Farooq P. Agha, ${ }^{1}$ Mark B. Orringer, ${ }^{2}$ and Marco A. Amendola ${ }^{1}$ \\ Departments of ${ }^{1}$ Radiology and ${ }^{2}$ Surgery, Section of Thoracic Surgery, The University of Michigan Hospitals, Ann Arbor, \\ Michigan, USA
}

\begin{abstract}
Gastric interposition was achieved in 138 patients following transhiatal esophagectomy without thoracotomy. Among these, 33 had benign and 105 malignant lesions. All patients were evaluated on the 10th postoperative day with a barium swallow examination. However, if an anastomotic leak was suspected clinically before this time, a water-soluble contrast study was initially obtained. Early postoperative complications included anastomotic leaks (15), cricopharyngeal incoordination with aspiration (6), and gastric perforation (2). Late postoperative complications included anastomotic strictures (12), pyloric stenosis (4), recurrence of tumor (3), and transhiatal visceral herniation (2). Our technique of postoperative radiographic evaluation, particularly when a leak is suspected clinically, is discussed.
\end{abstract}

Key words: Esophagus, surgery - Esophagectomy, complications - Gastric interposition, radiography.

The relative efficacy of various techniques of visceral esophageal substitution for the obstructed or malfunctioning esophagus has long been the subject of controversy among surgeons. The most common alternatives include a skin tube (Bircher, 1894); the jejunum (Roux and Herzen, 1907); colon (Kelling and Vulliet, 1911); and stomach (Kirschner, 1920) [1]. Because of its resilience, ability to reach to the pharynx, and relative technical advantages, the stomach has now emerged as the organ most frequently utilized to replace the

Address reprint requests to: Farooq P. Agha, M.D., Department of Radiology, Box 013, The University of Michigan Hospitals, Ann Arbor, MI 48109, USA esophagus in both benign and malignant disease [2-16]. Since esophagectomy for carcinoma is performed primarily for palliation, neither local tumor extension nor metastatic adenopathy is an absolute contraindication to surgery. Direct invasion of the major mediastinal vessels or the tracheobronchial tree or the presence of extensive distant metastases commonly precludes operation. In patients with benign esophageal lesions, gastric interposition may be used for treatment of strictures refractory to dilatation, recurrent esophagitis following failed antireflux operations, or neuromuscular disorders of the esophagus (achalasia or spasm) refractory to esophagomyotomy.

Note only does the technique of transhiatal esophagectomy without thoracotomy avoid the morbidity of a thoracic incision in these patients, but also the cervical esophageal anastomosis used eliminates the potential hazard of mediastinitis and sepsis resulting from an intrathoracic anastomotic leak. We report our experience with the radiologic evaluation of visceral esophageal substitution using stomach in 138 patients following transhiatal esophagectomy. This report describes the distinctive postoperative radiographic features of gastric interposition and the most common radiologically detectable complications.

\section{Materials and Methods}

Between January 1977 and December 1982, transhiatal esophagectomy (THE) with gastric interposition was performed in 138 patients as described previously $[3,22]$. One hundred and five patients, including 75 men and 30 women ranging in age from 39 to 92 years (average, 64), underwent esophagectomy for malignant disease of the esophagus. The malignant lesions treated consisted of primary squamous cell carcinoma (68 patients), adenocarcinoma of esophagus and gastroesophageal junction (10 patients), adenocarcinoma arising in Barrett's esophagus (19 patients), pharyngeal carcinoma (5 patients), and diffuse anaplastic carcinoma of the entire thoracic esophagus, 

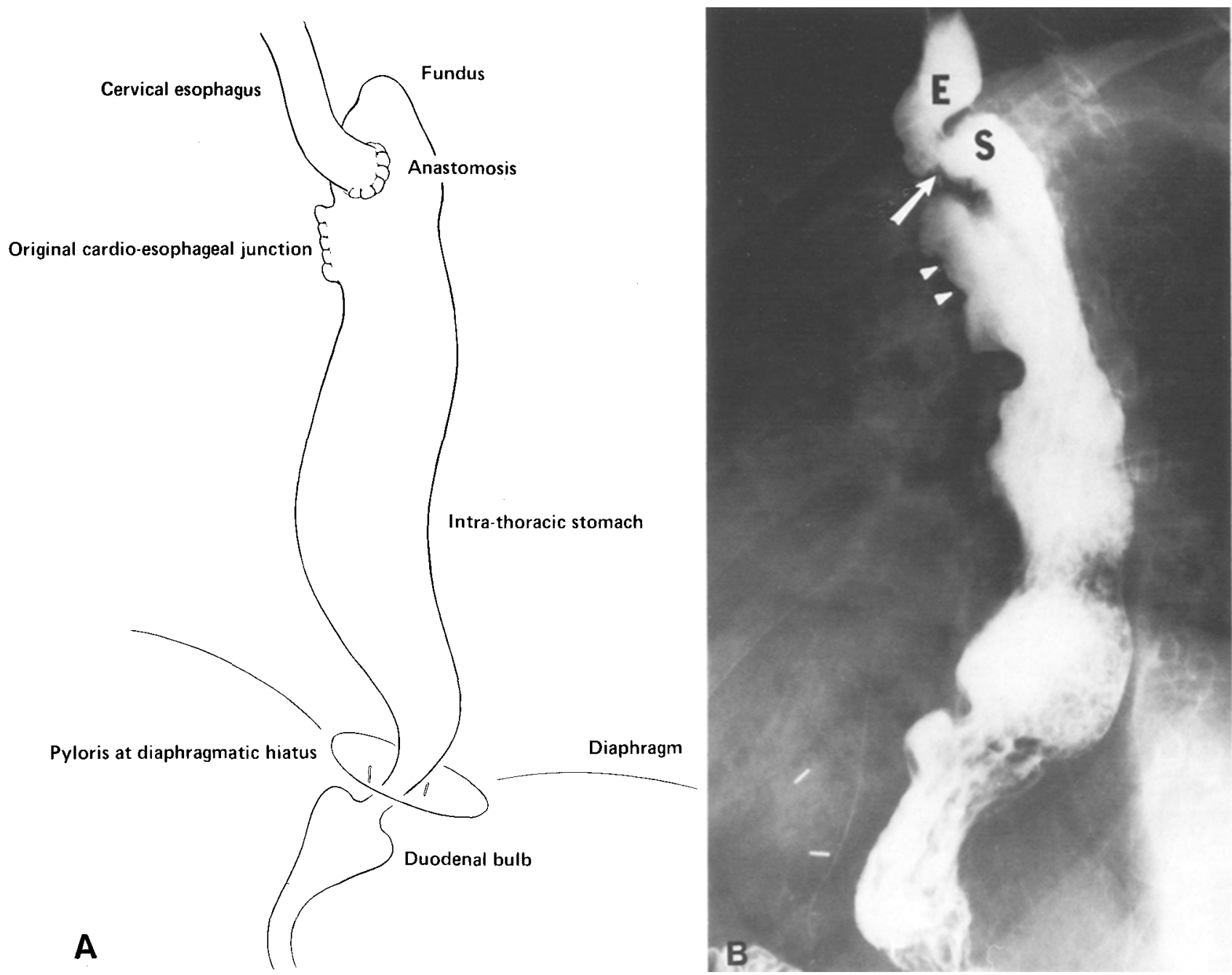

Fig. 1. A Schematic drawing of anatomy following cervical esophagogastrostomy and gastric interposition in the posterior mediastinum. B Normal postoperative appearance of transposed intrathoracic stomach. Barium swallow in slightly right posterior oblique projection shows end-to-side cervical esophagogastric anastomosis (arrow) marked by clips. Part of gastric fundus $(S)$ is seen above and behind the anastomosis (pseudodiverticular appearance). Site of closure of original gastroesophageal junction (arrowheads) may simulate a gastric ulcer (pseudoulcer). $E$, esophagus

metastatic involvement of cervical esophagus by thyroid carcinoma, and squamous cell carcinoma of trachea invading the esophagus, each in 1 patient. In 5 patients, stomach was positioned substernally in the anterior mediastinum, and in 100 patients, in the posterior mediastinum in the original esophageal bed. Among 33 patients undergoing esophagectomy for benign disorders of the esophagus, there were 9 men and 24 women ranging in age from 18 to 87 years, with a mean age of 42 years. The benign conditions treated consisted of peptic strictures not corrected by antireflux operations (9), caustic-induced strictures (5), neuromuscular esophageal disorders (11) (achalasia in 6, diffuse esophageal spasm in 5), Barrett's strictures (3), scleroderma with peptic stricture (2), and perforated esophagus, monilia-induced stricture, and postemetic stricture (1 each).

\section{Surgical Technique of Transhiatal Esophagectomy}

The peritoneal cavity is entered through an upper midline abdominal incision, and the stomach is mobilized, preserving the right gastric and gastroepiploic vessels. The gastroesophageal junction is encircled, and the thoracic esophagus is bluntly dissected from the posterior mediastinum to the level of the carina by a hand inserted through the diaphragmatic hiatus. The cervical esophagus is encircled through an oblique left cervical incision and the plane between the upper trachea and the esophagus is developed by finger dissection into the superior mediastinum. The entire intrathoracic esophagus is mobilized by 1 hand working upward from the diaphragmatic hiatus and the other working downward from the cervical incision. The cervical esophagus and gastroesophageal junction are divided, and the thoracic esophagus is removed from the field. The stapled gastric end of the divided cardia is oversewn. The entire hand and forearm are passed through the diaphragmatic esophageal hiatus and posterior mediastinum until 3 or 4 fingers are visible in the cervical incision. This insures an adequate mediastinal tunnel for the visceral esophageal substitute. A pyloromyotomy is then performed to avoid gastric emptying problems following the vagotomy which inevitably accompanies the esophagec- 

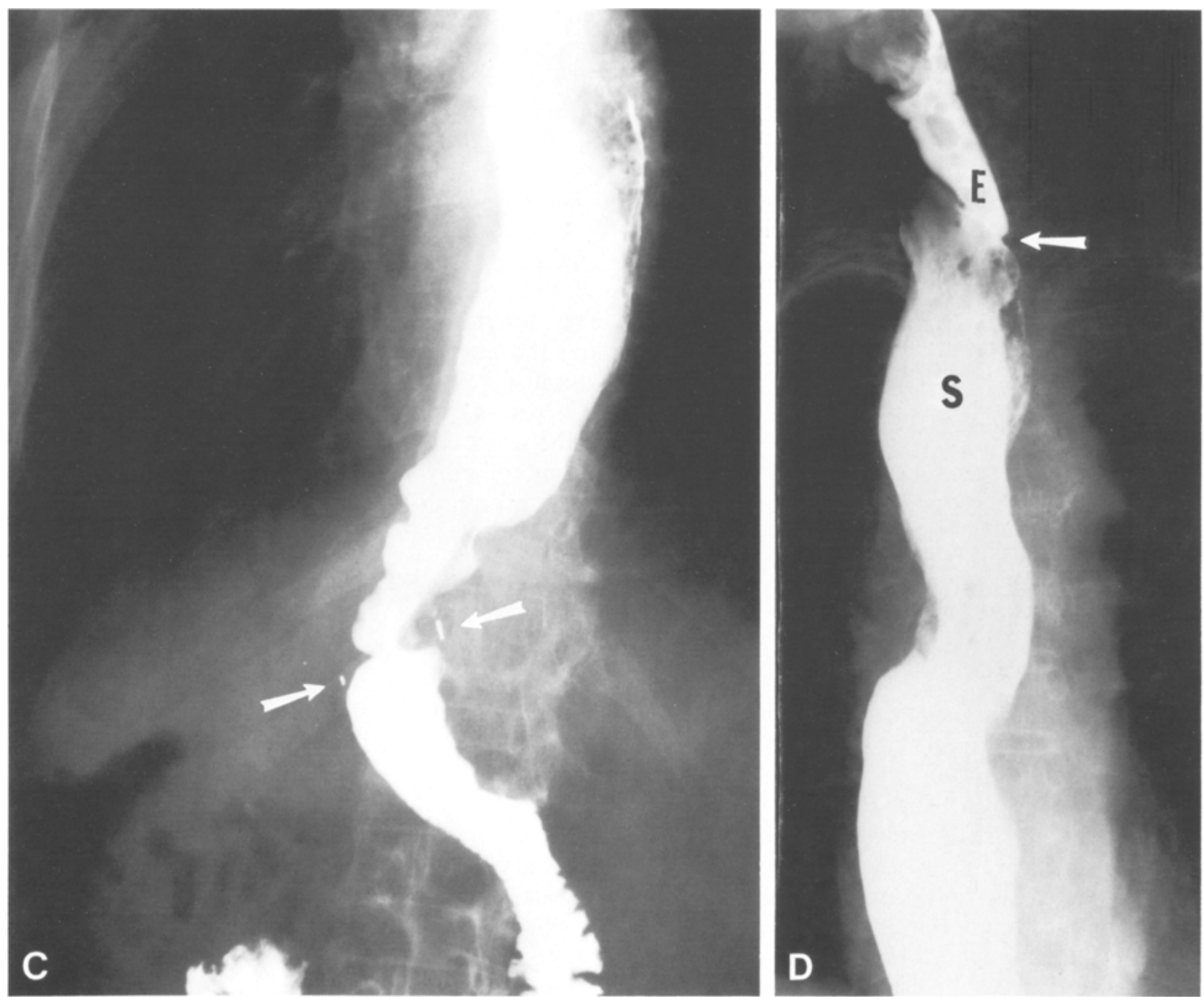

Fig. 1. C Distal part of the stomach: the pylorus is at the diaphragmatic hiatus with duodenal bulb below the diaphragm. Metallic clips mark the site of pyloromyotomy (arrows). D Anteroposterior view shows angular appearance of the cervical esophagogastric anastomosis seen above the level of the clavicles (arrow). Most of the proximal stomach $(S)$ is seen to the right of the midline and returns to the left above the diaphragmatic hiatus. In the late postoperative period, stomach becomes "esophagized," folds assume a longitudinal configuration, and the calibre of the stomach decreases. $E$, esophagus

tomy. The stomach is then transposed through the diaphragmatic hiatus and into the posterior mediastinum until the fundus appears in the cervical incision. The gastric fundus is then sutured to the prevertebral fascia, and an end-to-side anastomosis between the cervical esophagus and gastric fundus is performed. Postoperative decompression of the intrathoracic stomach is achieved with either a pharyngostomy or nasogastric tube. A feeding jejunostomy is inserted routinely in all of our patients undergoing esophageal substitution, so that in the event of an anastomotic leak, the gastrointestinal tract can still be utilized for alimentation [3, 22].

\section{Technique of Radiologic Examination}

All patients were evaluated on the 10 th postoperative day with a barium swallow. A conventional single-, rather than a doublecontrast study, was preferred, since effervescent granules used in double-contrast examination may overdistend the intratho- racic stomach and produce undesirable side effects and tension at the anastomotic site. Low-density barium sulfate mixture ( $40 \%$ weight by weight) was used for the single-contrast examination. Fluoroscopic spot films of the upper anastomosis and pylorus, as well as overhead films $(35.5 \times 43 \mathrm{~cm})$ in anteroposterior and lateral positions, were obtained. An upright film was routinely obtained at $20-30$ minutes to evaluate the emptying dynamics of the interposed stomach. Follow-up barium swallows were performed to evaluate symptoms of dysphagia or regurgitation.

When an anastomotic leak was suspected clinically because of the development of an otherwise unexplained postoperative fever, the patient was examined initially with water-soluble contrast. The patient was placed supine or in a slightly right posterior oblique position with the $\mathrm{x}$-ray table in $30-45^{\circ}$ reversed Trendelenburg position to prevent aspiration into the tracheobronchial tree. When an initial water-soluble contrast study showed no leak, the patient was asked to drink the low-density barium sulfate mixture and spot films were repeated. 
Table 1. Radiologically detectable complications of gastric interposition after transhiatal esophagectomy in 138 patients

\begin{tabular}{ll}
\hline Complications & $\begin{array}{l}\text { No. of } \\
\text { patients }\end{array}$ \\
\hline
\end{tabular}

Early (within 30 days of operation):

Anastomotic leak

Pharyngogastric

Cervical esophagogastric

Cricopharyngeal incoordination with aspiration

Gastric perforation

15

9

6

6

2

Late ( 30 days or more after operation):

Anastomotic stricture

(10 treated with dilatations;

2 required surgical revision)

Pyloric stenosis

Recurrence of tumor

Transhiatal visceral herniation

transverse colon (1), jejunum (1)

\section{Results}

\section{Normal Radiologic Features}

The cervical esophagogastric anastomotic site is located at or above the level of the clavicles and is identifiable radiographically by 2 radiopaque metallic clips placed at the time of operation (Fig. 1). The angular appearance of the anastomosis is due to its end-to-side construction, with the cervical esophagus above the anastomosis showing a slightly oblique course slanting to the left of the midline. Because at operation the gastric fundus is suspended from the prevertebral fascia prior to beginning the anastomosis on the anterior gastric wall, radiographically a portion of the fundus is seen above and behind the anastomotic site. This may produce a pseudodiverticular or saccular appearance that could be confused with gross anastomotic disruption (Fig. 1). The site of closure of the original esophagogastric junction may simulate a gastric ulcer ("pseudoulcer" appearance of closed cardia) (Fig. 1). The presence of surgical staples in this area of irregularity, however, indicates the origin of the radiographic appearance. The proximal part of the body of the stomach is projected to the right of the midline and gradually returns to the left distally. The pylorus is seen at the level of the diaphragmatic hiatus and is indicated by 2 radiopaque metallic clips. The duodenal bulb is identified below the diaphragm. In the immediate postoperative period, the pylorus may appear narrow; however, the transit of barium through it is usually satisfactory. Initially after operation, the intrathoracic stomach is atonic or slightly dilated, and its borders appear smooth and parallel. The passage of barium across the cervical anastomosis and through the stomach into the duodenum is generally unimpeded. This hypotonia of the stomach usually subsides within 3-4 weeks.

In the late postoperative period the anastomotic site appears pliable, without any mucosal edema or nodular indentations. The stomach tends to adopt more of an esophageal configuration: the mucosal folds become more parallel and the lumen width diminishes, producing a tubular appearance. The gastric and duodenal hypotonia disappears and the gastric emptying becomes normal. Occasionally previous gastric surgery (gastric resection, gastrostomy, etc.) may produce a contour defect in the stomach wall. Knowledge of such prior operations will prevent potential diagnostic pitfalls during interpretation.

\section{Complications}

The radiographic abnormalities identified within the first 30 days of operation have been termed early and those after 1 month, late. These are summarized in Table 1 . During the early postoperative period, 3 abnormalities have been encountered: anastomotic leak, tracheobronchial aspiration, and gastric perforation. In the present series, of 15 anastomotic leaks identified, $9(30 \%)$ were among 30 pharyngogastric and $6(5.5 \%)$ were among 108 cervical esophagogastric anastomoses (Fig. 2). In 3 patients, anastomotic leaks not detected with water-soluble contrast agent were demonstrated using barium. Mild transient cricopharyngeal incoordination is common in the early postoperative period, and in 6 patients, pharyngeal incoordination was severe enough to cause recurrent aspiration into the tracheobronchial tree. Two patients developed gastric perforations. The first had systemic lupus erythematosus being treated with steroids and underwent esophagectomy and gastric interposition for recurrent peptic strictures. She experienced perforation of her intrathoracic stomach on the 12th postoperative day and required emergency surgical repair (Fig. 3). The second patient developed a cervical gastric leak at the site of suspension of the gastric fundus from the prevertebral fascia.

The late postoperative complications consisted of anastomotic strictures, pyloric stenosis, tumor recurrence, and transhiatal visceral herniation. Among the 12 patients who developed late postoperative anastomotic strictures, 10 were treated successfully with frequent dilatations, and 2 required surgical revision. Six patients who experienced postoperative anastomotic leaks subse- 

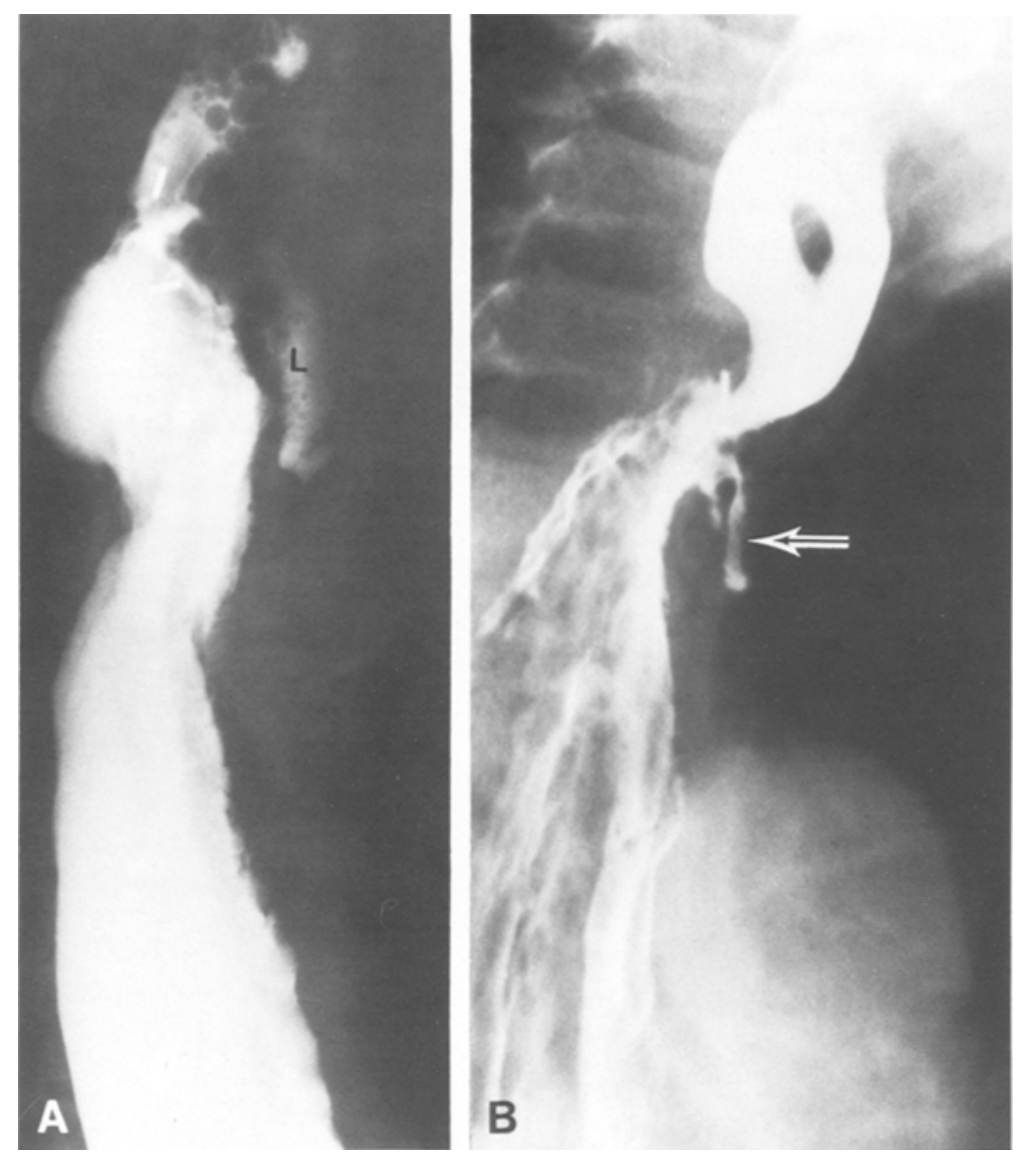

Fig. 2. Cervical esophagogastric anastomotic leaks. A Demonstration of a leak $(L)$ in the left side of the neck with water-soluble medium. B This small anastomotic leak (arrow) seen on barium swallow was not visible during initial evaluation with iodinated contrast material
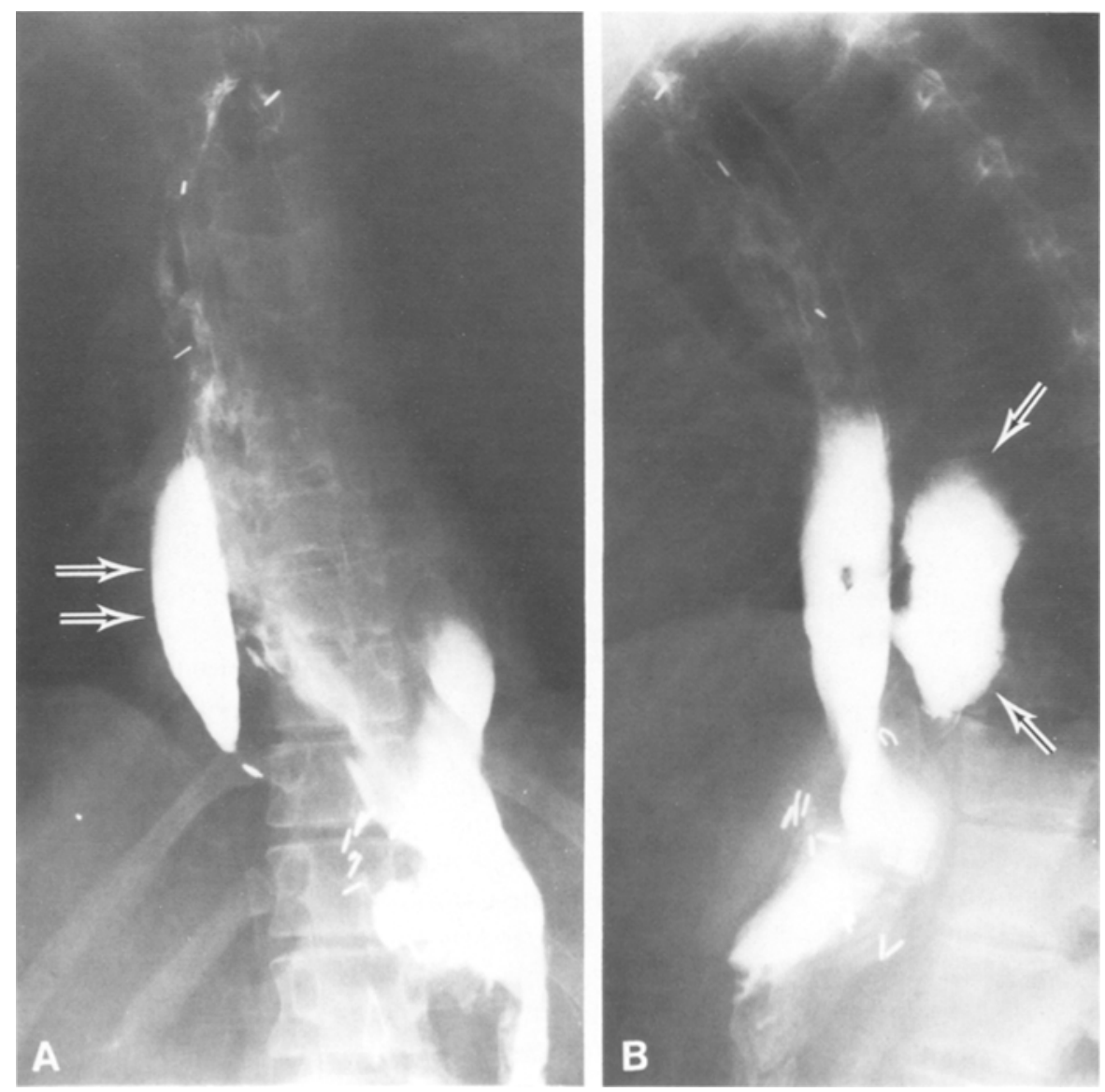

Fig. 3. Gastric perforation. A, B Radiographs show linear collection of aqueous contrast material in the mediastinum (arrows) from gastric perforation occurring on the 12 th day after cervical esophagogastrostomy. This patient had systemic lupus erythematosus and was on steroid medications 

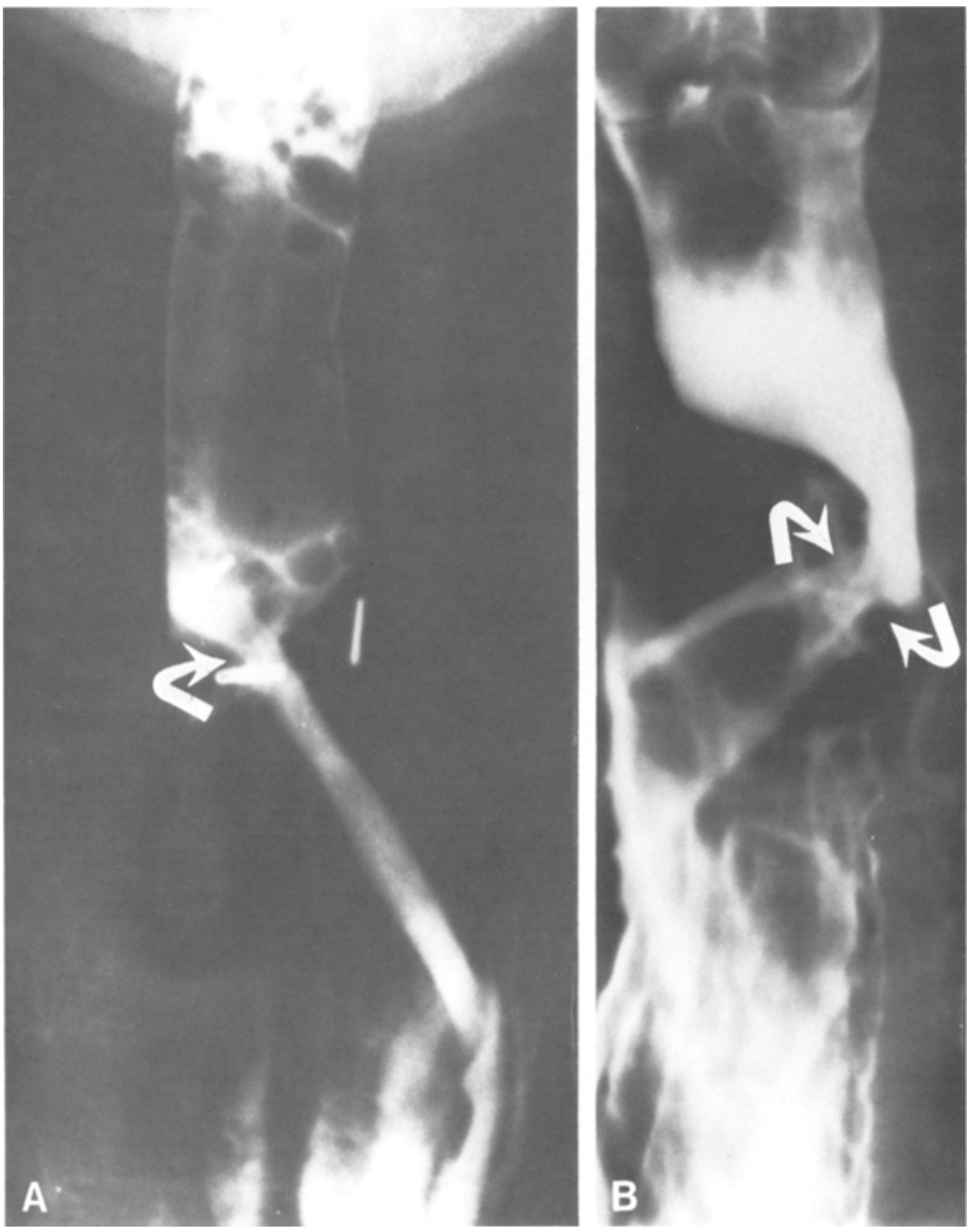

Fig. 4. Cervical esophagogastric anastomotic strictures. A, B Barium "esophagograms" in 2 different patients show cervical esophagogastric strictures. An esophageal "jet phenomenon" is seen with greater frequency as the narrowing of the stricture site progresses (curved arrows) quently developed strictures. Radiologically strictures demonstrate a barium "jet phenomenon," thus providing a means of calibrating their diameter (Fig. 4). Pyloric stenosis developed in 4 patients (Fig. 5) and responded to dilatation under fluoroscopic control. In 1 patient, the pyloric stenosis was due to recurrence of tumor at the juxtadiaphragmatic region. Recurrent carcinoma may result in distortion of any portion of the interposed stomach. Three of our patients developed local recurrence, manifested as cervical esophagogastric anastomotic stenosis in 1, pyloric stenosis in 1, and direct invasion of the intrathoracic stomach due to recurrence of large mediastinal tumor mass in the third (Fig. 6). Transhiatal visceral herniation alongside the interposed stomach occurred in 2 patients. A small segment of jejunum herniated alongside the substituted stomach in 1 patient 2 months following operation, and a segment of transverse colon in the 2 nd patient 5 years follow- ing operation. Both patients required surgical reduction of the herniated bowel back into the abdomen and narrowing of the diaphragmatic hiatus.

\section{Discussion}

Recently, the stomach has emerged as the organ of choice for visceral esophageal substitution, not only for malignant but also for a variety of benign esophageal lesions $[3,22,23]$. The most important complication of gastric interposition after transhiatal esophagectomy, as with all types of visceral esophageal substitution, is anastomotic leak, the incidence of which has been reported to range from 25 to $40 \%$ in different series [17-21]. A pharyngogastric anastomosis following laryngopharyngectomy is associated with a higher leak rate than a cervical - esophagogastric anastomosis, generally performed at or just above the level of the clavicles [22]. A pharyngeal anastomosis requires the maxi- 

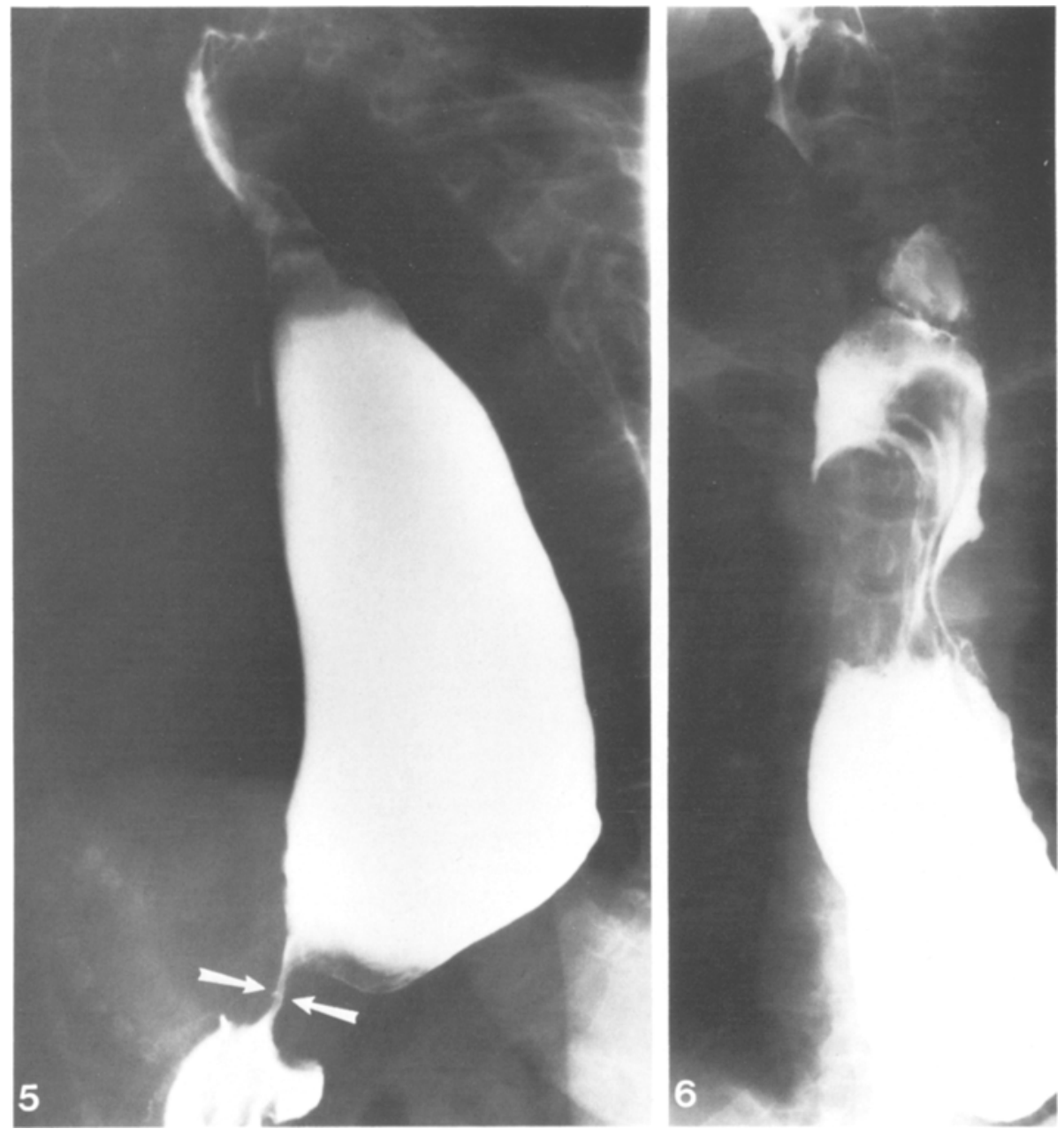

Fig. 5. Barium swallow 6 months after transhiatal esophagectomy and cervical esophagogastrostomy shows marked pyloric stenosis (arrows). Intrathoracic stomach is dilated and showed poor emptying

Fig. 6. Barium swallow 1 year after transhiatal esophagectomy and cervical esophagogastrostomy shows large mass invading the intrathoracic stomach due to recurrent mediastinal tumor

mum cephalad reach of the mobilized stomach, and tension on the anastomosis may contribute to the higher incidence of leakage. In addition, many patients requiring laryngopharyngectomy for carcinoma have undergone cervical radiation and wound healing is not normal because of radiation changes. The timely and accurate diagnosis of anastomotic disruption is critical, since the morbidity and mortality of an esophageal perforation are directly related to the interval between the diagnosis of the injury and institution of therapy.

The second most common complication of esophageal substitution is anastomotic stricture. An incidence of $10 \%$ has been found in most reported series $[2,15,18]$. The stricture is more likely to follow an anastomotic leak and is usually the late result of associated healing and fibrosis. We encountered $12(8.7 \%)$ strictures in this series.

About $20-25 \%$ of patients developed mild transient cricopharyngeal incoordination in the early postoperative period. This may be due to transient recurrent laryngeal nerve paresis from traction injury at surgery or to the effects of mobilization of the cervical esophagus during the operation [24]. Like the resulting hoarseness of the voice, the tendency to aspirate due to cricopharyngeal incoordination resolves within 4-6 weeks postoperatively. However, in 6 patients pharyngeal incoordination was severe enough to cause recurrent aspiration into the tracheobronchial tree. Extreme caution should be exercised when using water-soluble contrast, lest aspiration precipitate pulmonary edema. In the case of neoplastic disease, development of pharyngeal incoordination may signal local recurrence or metastatic disease to the brain affecting the vagal innervation of the cervical esophagus. Despite the pyloromyotomy performed to facilitate gastric emptying in the early postoperative period, the area of the pylorus may appear narrow. Gastric emptying in the upright position is usually satisfactory, however. Pyloric stenosis may develop as a late complication and usually 
responds to dilatation under fluoroscopic control. The diagnosis is suggested clinically by symptoms of vomiting and retrosternal fullness. Radiographically the intrathoracic stomach is distended and contains an excessive amount of secretions. There is marked delay in gastric emptying in the upright position.

Barium studies are helpful in evaluating malfunctioning of the intrathoracic stomach and distinguishing benign causes from tumor recurrence. The presence of a mass invading or indenting the interposed stomach anywhere along its intrathoracic course, nodular mucosal impressions, and eccentric narrowing of the lumen strongly suggest recurrent neoplasm.

Gastric interposition is an increasingly popular technique of replacing the esophagus when palliating esophageal cancer and in the definitive treatment of selected benign esophageal diseases. Familiarity with the distinctive radiologic features of gastric interposition and its complications will permit accurate assessment of postoperative complications and avoid potential diagnostic pitfalls.

Acknowledgments. The authors gratefully acknowledge the assistance of Professor Denis C. Lee, Department of Medical and Biological Illustration, for the schematic drawing used in Figure $1 \mathrm{~A}$ and Juanita Cantrell for secretarial assistance.

\section{References}

1. Storey CF: Acquired Surgical Lesions of the Esophagus. Springfield, Ill: Charles C. Thomas, 1962, pp 188-209

2. Postlethwait RW: Colonic interposition for esophageal substitution. Surg Gynecol Obstet 156:337-383, 1983

3. Orringer MB, Sloan H: Esophagectomy without thoracotomy. J Thorac Cardiovasc Surg 76:643-654, 1978

4. Denk W: Zur Radikaloperation des Oesophaguskarzinoms. Zentralbl Chir 40:1065-1068, 1913

5. Turner GG: Carcinoma of the esophagus - the question of its treatment by surgery. Lancet 1:67-72, 130-136, 1936

6. Ong GB, Lee JC: Pharyngogastric anastomosis after esophagopharyngectomy for carcinoma of the hypopharynx and cervical esophagus. Br J Surg 48:193-200, 1960

7. LeQuesne LP, Ranger R: Pharyngo laryngectomy with im- mediate pharyngogastric anastomosis. $B r \quad J \quad$ Surg $53: 105-109,1966$

8. Leonard JR, Maran AG: Reconstruction of the cervical esophagus via gastric anastomosis. Laryngoscopy $80: 849-862,1970$

9. Stell PM: Esophageal replacement by transposed stomach. Arch Otolaryngol 91:166-170, 1970

10. Akiyama H, Sato Y, Takahashi F: Immediate pharyngogastrostomy following total esophagectomy by blunt dissection. Jpn J Surg 225:3-56, 1971

11. Mappes $G$, Haas E: Closed transmediastinal relocation of the stomach for total esophageal replacement in one stage operation for carcinoma of the hypopharynx and cervical esophagus. Dtsch Med Wochenschr 100:826-828, 1975

12. Okada N, Tagami Y, Morishiti H: Reconstruction of the esophagus by posterior invagination esophagogastrostomy. World J Surg 1:361-370, 1977

13. Kirk RM: Palliative resection of esophageal carcinoma without formal thoracotomy. Br J Surg 61:689-690, 1974

14. Thomas AN, Dedo HH: Pharyngogastrostomy for treatment of severe caustic stricture of the pharynx and esophagus. J Thorac Cardiovasc Surg 73:817-824, 1977

15. Szentpetery S, Wolfgang T, Lower RR: Pull through esophagectomy without thoracotomy for esophageal carcinoma. Ann Thorac Surg 27:399-403, 1979

16. Agha FP, Orringer MB: Colonic interposition: radiographic evaluation. AJR 142:703-708, 1984

17. Huguier M, Gordin F, Maillard JN, Lortat-Jacob JL: Results of 117 esophageal replacements. Surg Gynecol Obstet 130:1054-1058, 1970

18. Ong GB: Resection and reconstruction of the esophagus. Curr Probl Surg 8:3-56, 1971

19. Hermreck AS, Crawford DG: The esophageal anastomotic leak. Am J Surg 132:794-798, 1976

20. Guidiceli R, Fuentes P, Rebound E: Surgical complications of esophageal surgery. Ann Anesthesiol Fr 18:373-376, 1977

21. Silver CE: Gastric pull up operation for replacement of cervical portion of esophagus. Surg Gynecol Obstet $142: 243-245,1976$

22. Orringer MB, Orringer JS: Esophagectomy without thoracotomy: a dangerous operation? J Thorac Cardiovasc Surg $85: 72-80,1983$

23. Orringer MB, Orringer JS: Esophagectomy: definitive treatment for esophageal neuromotor dysfunction. Ann Thorac Surg 34:237-248, 1982

24. Henderson RD, Boszko A, VanNostrand AWP: Pharyngoesophageal dysphagia and recurrent laryngeal nerve palsy. J Thorac Cardiovasc Surg 68:507-512, 1974

Received: February 23, 1984 ; accepted: May 8, 1984 